\title{
Numerical Analysis of Microwave Detection of Breast Tumours using Synthetic Focussing Techniques
}

\author{
R.Nilavalan ${ }^{1}$, J.Leendertz ${ }^{1,}$ I.J.Craddock*2, A. Preece ${ }^{\text {, }}$, and R.Benjamin ${ }^{3}$ \\ ${ }^{\prime}$ Dept. of Medical Physics, University of Bristol, UK \\ ${ }^{2}$ Dept. of Electrical \& Electronic Engineering, University of Bristol, UK \\ ${ }^{3} 13$ Bellhouse Walk, Bristol, BSII OUE, UK
}

\section{Introduction}

Breast cancer is the most common cancer in women and second leading cause of female cancer mortality [1]. Currently X-ray mammography is widely used to detect breast cancer tumours [2], however it suffers from a relatively high missed- and false-detection rates and involves uncomfortable compression of the breast. X-rays are also ionising and therefore not generally suited to frequent screening.

Microwave detection of breast tumours is a non-ionising and potentially lowcost and more certain alternative. Analogous to Ground Penetrating Radars (GPRs), microwaves are transmitted employing an antenna array and the reflected signals, which contain reflections from tumours are recorded.

The work presented here employs a post reception synthetically focussed detection method developed for land mine detection [3]; all elements of an antenna array transmit a broadband signal in turn, the elements sharing a field of view with the current transmit element then record the received signal. By predicting the path delay between transmit and receive antennas via any desired point in the breast, it is then possible to extract and time-align all the signals from that point. Repeated for all points in the breast, this yields an image in which the distinct dielectric properties of malignant tissue are potentially visible.

This contribution presents a theoretical evaluation of the breast imaging system using FDTD methods. The FDTD model realistically models a practical system incorporating wide band antenna elements. One major challenge in Breast cancer detection using microwaves is the clutter arising from skin interface. Deeply located tumours can be detected using windowing techniques [4], however tumours closer to the skin interface require additional consideration, as described herein.

\section{The FDTD Model}

The FDTD model, shown in Figure 1, includes the array, and realistic representations of the skin, healthy breast tissue and the tumour. The antenna array consists of 16 wideband stacked patch elements designed for radiation into human breast tissue. The patch elements are coupled, via centrally placed slots in the antenna back plane, to microstrip feeds. The antenna elements have a $-10 \mathrm{~dB}$ feed-antenna match from 4 to $9 \mathrm{GHz}$ and have a satisfactory radiation pattern over this range [5]. A matching medium with dielectric properties similar to breast tissues was used between the array and the skin. 
These initial numerical experiments assume a flat skin consisting of a $1 \mathrm{~mm}$ thick dielectric with $\varepsilon_{\mathrm{r}}=40+j 5.86$. The tumour, with $2 \mathrm{~mm}$ diameter and $\varepsilon_{\mathrm{r}}=$ $50+\mathrm{j} 1.02$, is $10 \mathrm{~mm}$ from the skin.

The antenna elements are excited in tum using a Gaussian-modulated twocycle pulse at $6.5 \mathrm{GHz}$ and all non-excited elements record the received signals. 15 antenna elements were excited in separate FDTD runs to collect data for all possible unique paths. The signals recorded on the microstrip feeds consist of mutual coupling between antenna elements, signals reflected from skin and reflections from the tumour.

The mutual coupling between antenna elements can be eliminated by performing background subtraction without skin and tumour (possible in practice by using a phantom). Separating the small tumour reflections from the clutter due to skin is a considerably more challenging task.

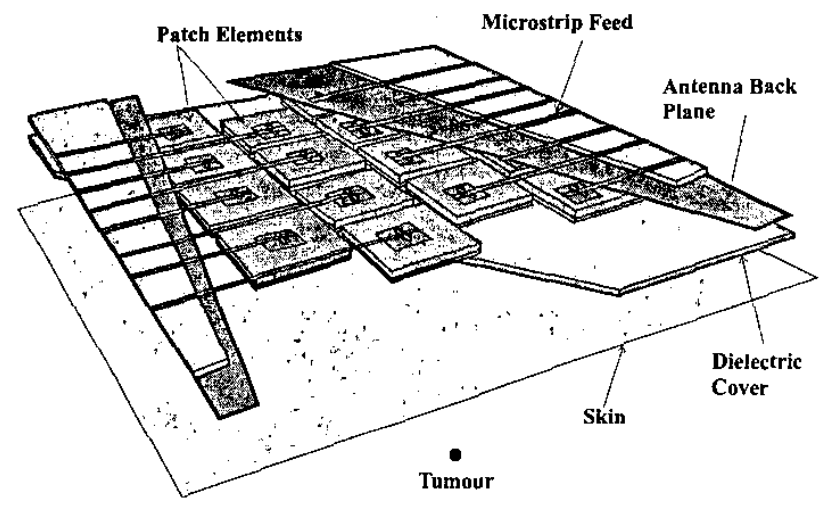

Figure 1 FDTD Antenna Array Model

Scanning of the breast tissue is performed by synthetically focussing over a 3D grid of points below the skin. The initial pre-processing of the recorded signals involves elimination of mutual couplings by background subtraction and the synthetic focussing step is performed by adding up the time-aligned signals to produce the focussed image as in equation 1.

$$
V=\int_{0}^{\tau}\left(\sum_{i=1}^{N} w_{i} U_{i}\left(t-T_{i}\right)\right)^{2} d t
$$

- where $U_{i}(t)$ is the signal received from path $i, N$ is the number of paths associated with the resolution cell, $w_{i}$ is a weighting factor for the attenuation, spreading losses along path $i$, and $T_{j}$ is the path delay for the trip to and back from the resolution cell (knowledge of this path delay requires estimation of the signal propagation velocity in the medium). As shown above, the retum associated with the any point of interest is computed by integrating the power in the sum of time-aligned and weighted signals, over a window corresponding to the transmit pulse width. 


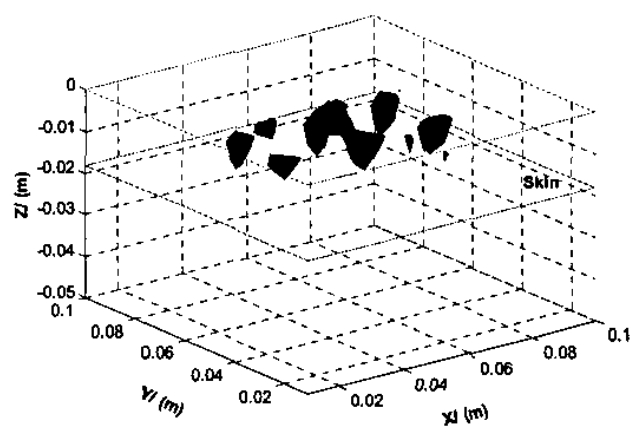

Figure 2 Three dimensional image with total signals

Figure 2 shows the focussed results without employing skin clutter removal techniques. It is obvious from this result that the skin reflection dominates and completely masks the signals from the tumour.

A reduction in these strong skin reflections can be obtained by recording the reflections at a slightly offset array location. Data collected at an offset location will contain approximately the same skin clutter but signals from the tumour will undergo phase and amplitude changes. Subtraction of these two sets of data and subsequent focussing will indicate a positive sum of signals at the tumour location and a negative sum at the offset location.

Figure 3 and Figure 4 clearly show the target at its correct location, without obscuration by the skin, after focussing the subtracted data, as explained above, and filtering out the positive signals.

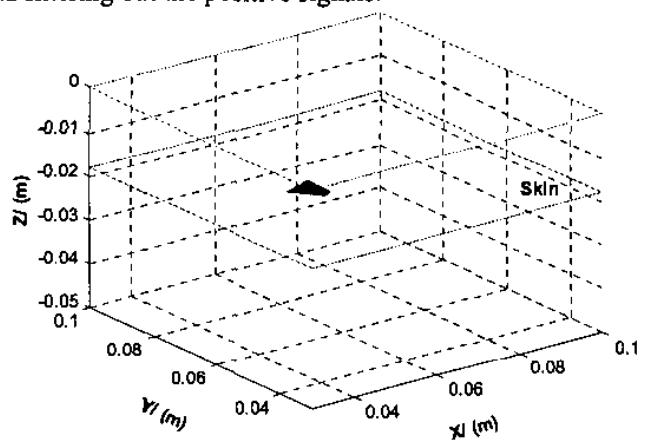

Figure 3 Three dimensional image with offset subtraction 


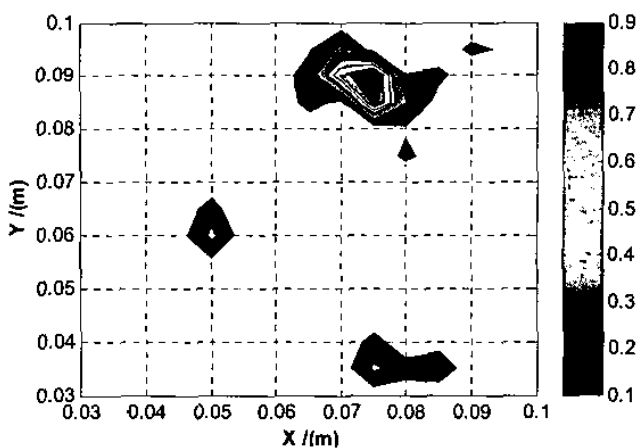

Figure 4 Horizontal Slice at target location

\section{Conclusions}

Initial results have been presented using a synthetically focussed radar approach to microwave imaging of the human breast. These initial results, derived using data from a realistic FDTD model, demonstrate successful detection of a small tumour in a lossy human breast. A technique to remove skin clutter has also been proposed and evaluated using numerical results.

The practical implementation of this approach, employing realistic phantoms [6], is in progress and practical results will be available shortly.

\section{References}

[1] A.G. R. Lynn, L. Carol, B. Kosary and F. Hankey, SEER cancer statistics review 1973-1994, National Cancer Inst., Bethesda, MD, NIH Pub, 2789, 1997.

[2] M. Brown, F. Houn, E. Sickles and L. Kessler, Screening Mammography in Community Practice, Amer. J. Roentgen, Vol. 165, pp 1373-1377, Dec 1995.

[3] R. Benjamin, I. J. Craddock, G. S. Hilton, S. Litobarski, E. McCutcheon, R. Nilavalan, G. N. Crisp, Microwave detection of buried mines using noncontact, synthetic near-field focusing. IEE Proceedings: Radar, Sonar \& Navigation, vol.148, no.4, Aug. 2001, pp.233-40.

[4] R. Nilavalan, A. Gbedemah, I. J. Craddock, X. Li and S. C. Hagness, Numerical Investigation of Breast Tumour Detection using Multi-Static Radar, Electronics Letters, vol. 39, Dec 2003, pp 1787-1789.

[5] R. Nilavalan, I. J. Craddock, A. Preece, J. Leendertz, R. Benjamin, "Breast Cancer Tumour Detection using Microwave Radar Techniques" Submitted to 2004 URSI EMT-S Symposium, Písa, 2004.

[6] J. Leendertz, A. Preece, R. Nilavalan, I. J. Craddock, R. Benjamin, "A Liquid Phantom Medium For Microwave Breast Imaging", 6th Congress of European Bioelectromagnetics Association (EBEA), 13-15 Nov 2003, Budapest, Hungary. 